\title{
Alveolar Ridge Preservation Using Autogenous Dentin Graft versus Demineralized Freeze-dried Bone Allograft (DFDBA): A Case Report
}

\author{
César Esquivel-Chirino, Vanessa Vargas-Romero, Gerardo Rodríguez-Torres, \\ Verónica Villatoro-Ugalde, María Guadalupe Rivas-Fonseca, Delina G. Montes-Sánchez, \\ Daniela Carmona Ruiz, Yolanda Valero Princet and Christian Márquez-Correa
}

\section{ABSTRACT}

Tooth extraction is a surgical procedure that is performed due to pulp and periapical pathology, periodontal disease, or teeth that are not deemed to be prosthetically or endodontically restorable. Following tooth extraction, bone resorption and vertical or horizontal defects occur. Traditionally, ridge preservation warrants the use of different agents such as autografts, allografts, xenografts, and mineral or ceramic materials. Autogenous dentin grafts are alternative to ridge preservation with osteoconductive, osteoinductive, and non-immunogenic properties to generate bone formation. The aim of the case report was to evaluate and compare clinical and histological outcomes using an autogenous dentin graft versus an allogeneic bone graft (DFDBA) as an alternative for ridge preservation.

Keywords: alveolar ridge preservation, DFDBA, autogenous dentin graft, tooth extraction.
Published Online: April 26, 2021

ISSN: $2684-4443$

DOI: $10.24018 /$ ejdent.2021.2.2.45

C. Esquivel-Chirino*

Area de Básicas Médicas, División de Estudios Profesionales, Facultad de Odontología, Universidad Nacional Autónoma de México, CDMX, Mexico.

(email: investigaciondental@gmail.com) V. Vargas-Romero

Especialidad de Periodoncia, Posgrado de la Facultad de Odontología, Universidad Intercontinental, CDMX, Mexico.

G. Rodríguez-Torres

Especialidad de Periodoncia, Posgrado de la Facultad de Odontología, Universidad Intercontinental, CDMX, Mexico.

V. Villatoro-Ugalde

Profesora del Posgrado y de la Facultad de Odontología, Universidad Intercontinental, CDMX, Mexico.

(e-mail: villatorovero@yahoo.com.mx) G. Rivas-Fonseca

Profesora de la Especialidad de Periodoncia, Posgrado de la Facultad de Odontología, Universidad Intercontinental, CDMX, Mexico.

(e-mail: mgperivasf@gmail.com)

D. Montes-Sánchez

Investigación Biomédica Básica.

Licenciatura en Estomatología.

Benemérita Universidad Autónoma de Puebla Complejo Regional Sur-Tehuacan, Puebla México, Mexico.

(e-mail: dely.ms ${ }^{@}$ gmail.com)

D. Carmona Ruiz

Area de Ortodoncia, División de Estudios Profesionales, Facultad de Odontología, Universidad Nacional Autónoma de México, CDMX, Mexico.

(email: danyelacarmona ${ }^{@}$ gmail.com)

Y. Valero Princet

Facultad de Odontología, División de la Salud, Universidad Intercontinental, Ciudad de Mexico, Mexico.

(email: mvalero@uic.edu.mx)

C. Márquez-Correa

Coordinador Especialidad de Periodoncia, Posgrado de la Facultad de Odontología, Universidad Intercontinental, CDMX, Mexico.

(e-mail: dr.christian.marquez ${ }^{\circledR}$ gmail.com)

*Corresponding Author 


\section{INTRODUCTION}

Tooth extraction is a surgical procedure to eliminate a tooth from its alveolar housing. It is conducted in cases in which a tooth is not endodontically or prosthetically restorable and in the presence of periodontal disease and periapical pathology. Following tooth extraction, cortical and alveolar bone undergo resorption, and vertical and horizontal bone defects can occur [1].

Schropp reported that bone resorption occurs after tooth extraction [2]. Tan and colleagues reported that a range of vertical bone loss between $11 \%$ and $22 \%$ and that of horizontal bone loss between $29 \%$ and $63 \%$ occurs 6 months after tooth extraction [3]. Bone defects reduce alveolar volume and hinder prosthesis retention as well as implant placement. During tooth extraction, ridge preservation is required to maximize bone formation using biomaterials with osteoconductive, osteoinductive, and osteogenic properties [4], [5]. Autogenous grafts are ideal for the reconstruction of bone defects because of their histocompatibility and osteoinductive properties. However, the amount that is obtained is limited and these grafts possess a higher resorption rate under mechanical loads and soft tissue tension. Another major drawback is that these grafts require a donor site, which increases morbidity [6]. Allografts have osteoconductive and osteoinductive properties because they contain bone morphogenetic proteins (BMPs). Bone allografts are provided as mineralized or demineralized products, such as demineralized freeze-dried bone allografts (DFDBA). The latter is an alternative to autogenous grafts because it is readily available in various sizes and amounts, and they may be stored for long periods of time [7].

Recently, the use of dentin as an autogenous graft has been investigated. Kim and Urist reported on the bone formation using this graft [8]-[12]. Moreover, it exhibits osteoinductive properties and integration to new bone, thereby creating an ideal site for implant placement [13]-[15].

It is a collagenous material that releases growth factors such as BMPs, particularly BMP-2, which induces bone regeneration [16]. Kim and colleagues investigated the particle size of dentin grafts and related processing methods to achieve more efficient bone formation [17]. Binderman and colleagues proposed a novel protocol to obtain dentin grafts of various particle sizes ranging from 300 to $1200 \mu \mathrm{m}$ [18]. The aim of this case report was to evaluate the clinical and histological outcomes of using an autogenous dentin graft versus an allogeneic bone graft (DFDBA) as an alternative for ridge preservation.

\section{CASE PRESENTAtion}

\section{A. Patient Information}

A 53-year-old male patient was treated at the Periodontics Division of the Universidad Intercontinental. No allergies or systemic or chronic conditions were reported in his medical history. Clinical, radiographic, and periodontal assessments including a periodontal chart were conducted. A diagnosis of periodontitis with a generalized extension, Stage IV, and grade B were determined (Fig. 1 a, b, c, d).
The patient was informed of the available treatment alternatives, and he signed an informed consent form.
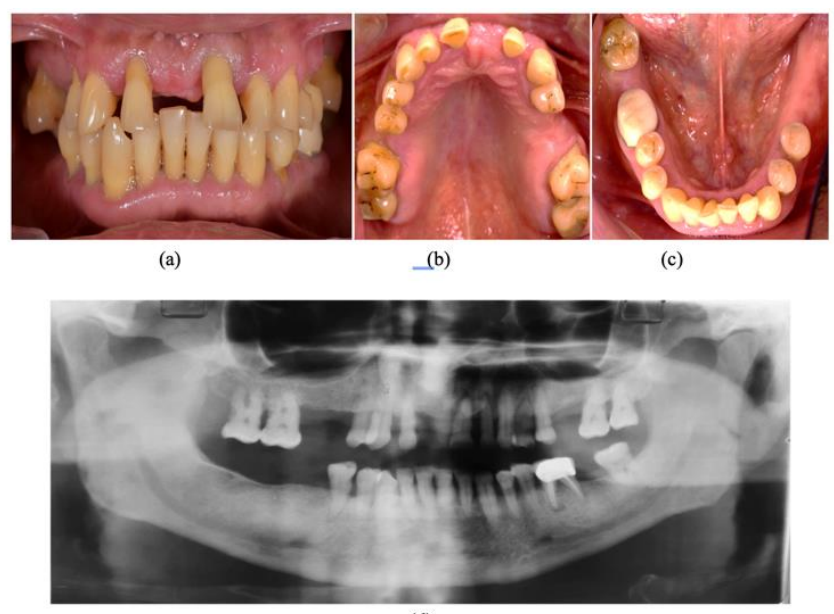

(d)

Fig. 1. Initial clinical images. (a) Occlusal view; (b) Intraoral upper (c) Intraoral lower; (d) Orthopantomography.

\section{B. Procedure}

Anesthesia included lidocaine $2 \%$ followed by the atraumatic extraction of the remaining 24 teeth (Fig. 2 a, b). Dentin autogenous graft processing was performed using the KometaBio ${ }^{\circledR}$ protocol, and teeth without previous root canal treatment, restorations, and pulp or periapical pathology were included [18].

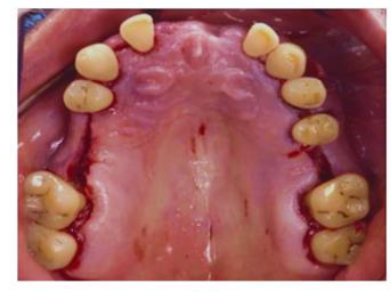

(a)

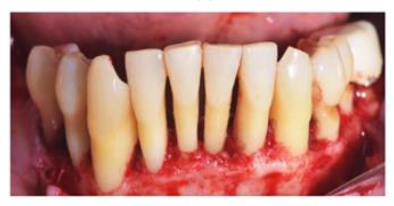

(c)

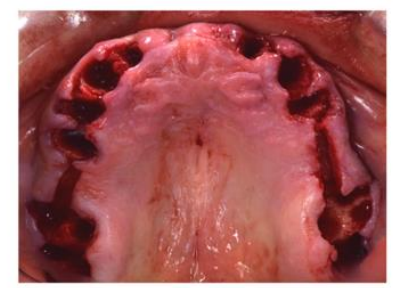

(b)

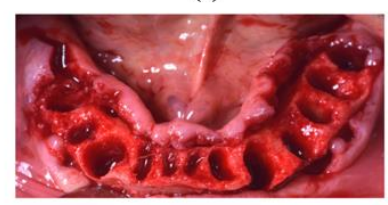

(d)
Fig. 2. (a) Teeth extraction upper; (b) clinical view after extraction upper; (c) teeth extraction lower; (d) clinical view after extraction lower.

\section{Protocol}

Extracted teeth were washed in saline (Fig. 3 a), and all restorative material was eliminated using rotary burs (Fig. 3 b). Teeth were triturated for 3 seconds using the Smart Dentin Grinder, and graft particles of 300-1200 $\mu \mathrm{m}$ were obtained, (Fig. 3 c, d, e). Particles were then submerged in $\mathrm{NaOH}$ solution $(0.5 \mathrm{M})$ and $20 \%$ alcohol for 10 minutes to eliminate organic material, bacteria, viruses, and fungi (Fig. $3 \mathrm{f}$ ).

The material was placed into a sterile buffer solution (PBS) with a $\mathrm{pH}$ of 7.5 (Fig. $3 \mathrm{~g}$ ). 


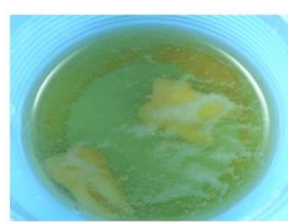

(a)

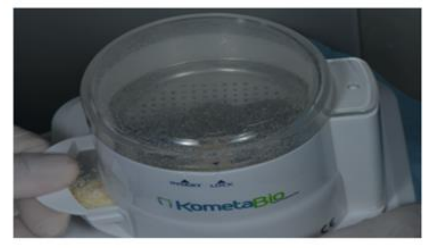

(d)

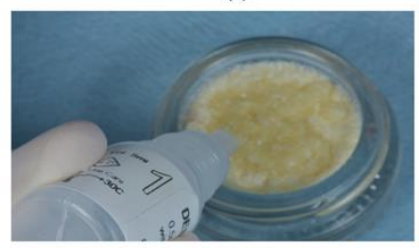

(f)

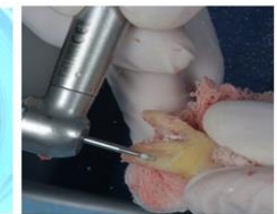

(b)

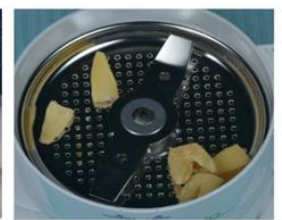

(c)

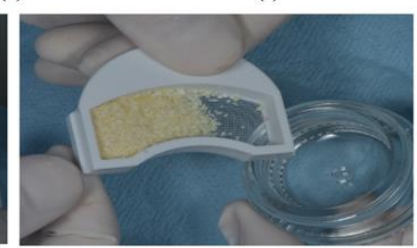

(e)

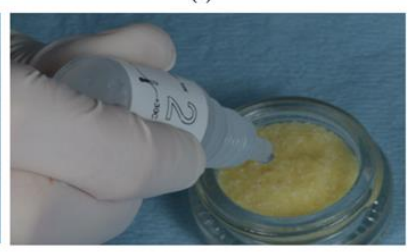

(g)
Fig. 3. (a) The teeth were immersed in saline solution; (b) Layers of enamel and cementum were removed; (c) Teeth were extracted inside the grinding chamber; (d) Smart dentin grinder chamber; (e) Tooth-sized particles were obtained from the Smart Dentin Grinder; (f) Dentin cleanser was used; (g) Phosphate buffered saline (PBS) was used.

\section{A ridge Preservation Procedure}

A ridge preservation procedure was performed using with a split-mouth design, in which a DFDBA bone graft (BioGraft ${ }^{\circledR}$ ) was placed in the right quadrant and autogenous dentin particles that were obtained using the KometaBio protocol (Fig. 4 d, e, f) were placed in the left quadrant. DFDBA particles in a sterile container were hydrated using saline and placed into the right-side maxillary and mandibular alveolar sockets (Fig. 4 a, b). A dentin autogenous graft was placed into the left maxillary and mandibular alveolar sockets (Fig. 4 d, e). To complete the surgical procedure, sutures (Vicryl 4-0) were placed to ensure hemostasis (Fig. 4 c, d).

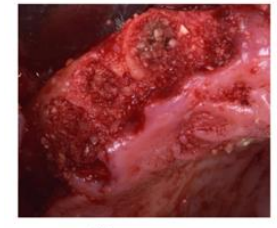

(a)

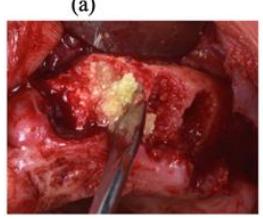

(d)

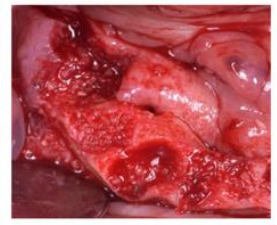

(b)

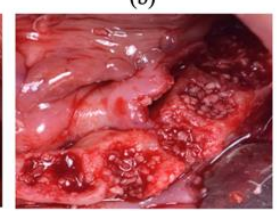

(e)

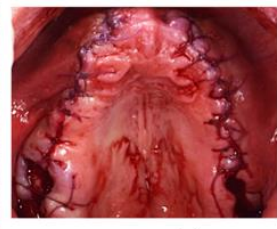

(c)

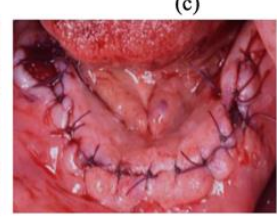

(f)
Fig. 4. Alveolar ridge preservation following post-extraction with allograft DFDBA on the right side (a) upper (b) and lower (c) clinical views after allograft placement. The autologous dentin graft on the left side (d) upper and (e) lower (f) clinical views after its placement.

\section{E. Tomography Evaluation}

Six months later, Cone Beam Computerized Tomography (CBCT) was obtained, and the Hounsfield scale (HU) was used to evaluate bone mineral density [19]-[20]. The maxillary and mandibular premolar areas were evaluated first bilaterally. Using DFDBA, a bone density of $585 \mathrm{HU}$ was observed in the maxillary right quadrant (Fig. 5 a) and $304 \mathrm{HU}$ in the mandibular right quadrant (Fig. 5 b).

In the maxillary left quadrant, a bone density of $583 \mathrm{HU}$ (Fig. 5 c) was observed with the dentin autogenous graft, and a density of $712 \mathrm{HU}$ was observed in the mandibular left quadrant (Fig. 5 d).

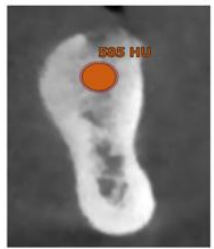

(a)

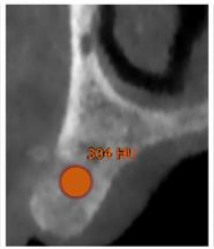

(b)

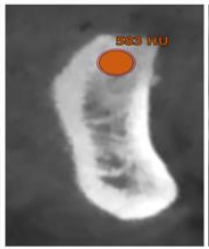

(c)

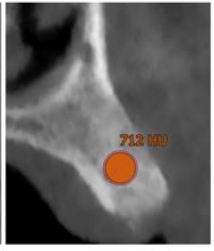

Fig. 5. Post-operative cone beam computed tomography (CBCT) after 6 months to analyze bone density. Allograft DFDBA in the (a) upper and (b) lower areas. Autologous dentin graft on the left side (c) lower and (d) upper areas in Hounsfield units (HU).

\section{F. Clinical Evaluation and Biopsy}

During this same study period, dentin and allogeneic grafts were clinically assessed. Healing of the soft tissues was observed with proper hydration, color, and texture and surgical re-entry was performed to obtain a biopsy of the grafted sites (Fig. 6 a, d). A trephine with a diameter of $2 \mathrm{~mm}$ was used to obtain the bone allograft (Fig. 6 b) and dentin autogenous graft samples (Fig. 6 e). This process was followed by $10 \%$ formalin fixation for 24 hours (Fig. 6 c, f). Samples were decalcified for 2 days and embedded in paraffin for histological analysis. Ground sections of $1 \mu \mathrm{m}$ were obtained and subsequently stained using hematoxylin and eosin $(\mathrm{H} \& \mathrm{E})$.

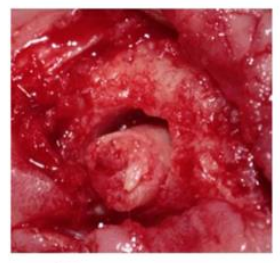

(a)

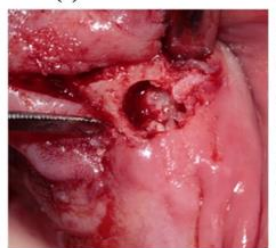

(d)

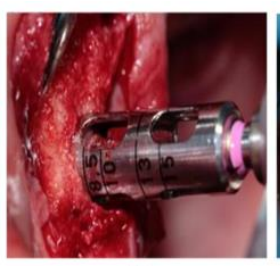

(b)

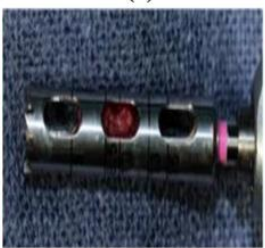

(e)

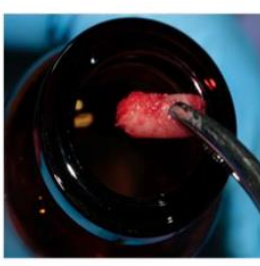

(c)

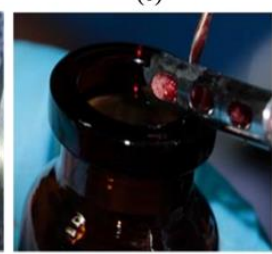

(f)
Fig. 6. (a) Clinical view after grafting of the allograft on the right side and the autologous dentin graft on the left side (d); (b) Sample obtained from bone grafted with allograft DFDBA on the right side and (e) the autologous dentin graft on the left side, $(\mathrm{c}, \mathrm{f})$ the tissue samples were formalin fixed for 24 hours

\section{G. Histological Analysis}

Histological analysis with $H \& E$ staining of DFDBA demonstrated formation of woven bone (WB) and new bone (NB) with the presence of osteocytes whit in lacunae and connective tissue (CT). Allograft particles were not observed (Fig. 7 a).

In the mineralized dentin graft sample that was stained with $\mathrm{H} \& \mathrm{E}$, some dentin particles (DT) were identified that were surrounded by new bone tissue (NB) with osteocytes within lacunae and connective tissue (CT) as well as some osteoblast 
activity (Fig. 7 c). Bone tissue samples that were stained with Masson's trichrome were analyzed with light microscope at $100 \times$ magnification (Fig. 7 b, d), and shows mature bone fusion with the new bone matrix that is surrounded by connective tissue and formation of new bone. Histological analysis of DFDBA demonstrated mature bone (WB) with the presence of a surrounding bone matrix $(\mathrm{BM})$ and connective tissue (CT) (Fig. 7 c). In the dentin autogenous graft sample at $100 \times$ magnification, we identified dentin particles (DT) that were characterized by dentinal tubules that show an association with the production of new bone matrix within them (embedding a dentin graft particle). Vascularized connective tissue is identified between bone spaces and dentin particles (DT).

Within the red box, an area that is characterized by incorporation and production of bone matrix (new bone) was observed between two mineralized dentin particles (Fig. 7 d).

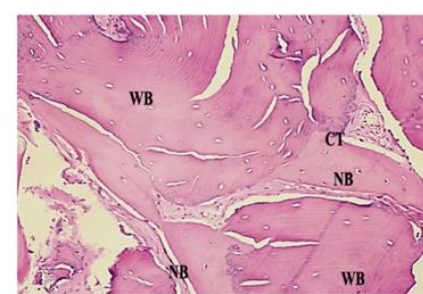

(a)

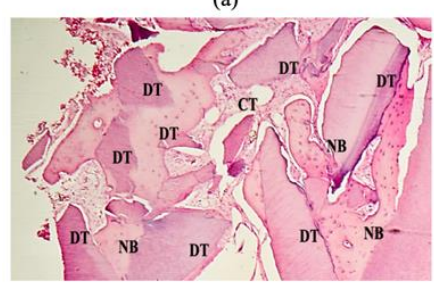

(c)

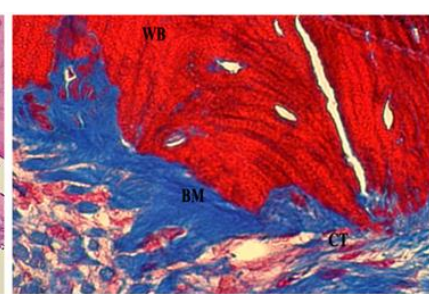

(b)

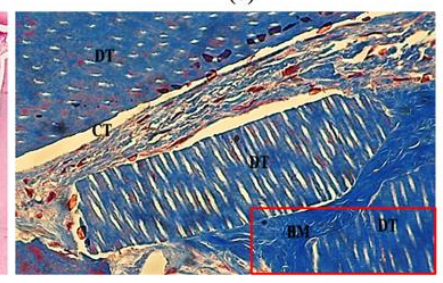

(d)
Fig 7. (a) Bone samples that were grafted using allograft DBDBA H\&E

$20 \times$ magnification show the presence of woven bone (WB) with bone

lacunae containing connective tissue (CT) new mineralized bone

formation $(\mathrm{NB})$ with the presence osteocytes within lacunae, and no residual allograft was detected; (b) Masson's trichrome stain shows bone samples grafted using allograft DBDBA $(100 \times$ magnification $)$ the presence of woven bone (WB) surrounding the bone matrix (BM); (c) Bone samples grafted using the autologous dentin graft show the presence of remaining

dentin particles (DT), which are surrounded by bone matrix (BM),

connective tissue (CT), and new bone formation that is in close contact

with dentin particles (NB); (d) Bone samples grafted with autologous dentin graft $(100 \times$ magnification) showing newly formed lamellar bone, the presence of the remaining dentin particles (DT) and connective tissue (CT).

\section{DISCUSSION}

Kim et al. reported that dentin lacks antigenicity and contributes to bone remodeling [8], and Kim and Urist investigated the usefulness of periodontally compromised teeth as an autogenous graft rather than as a biological waste [21]. Kim and colleagues stated that osteoconductive properties depend primarily on the dentin processing technique [8]-[12]. Another study published by Andrade et al. analyzed the histological and clinical results of a dentin block that contained a combination of autologous particulate dentin and leukocyte- and platelet-rich fibrin and liquid fibrinogen for alveolar ridge preservation. There were no side effects. Histological analysis after 6 months showed an increase in the amount of bone and that the bone was compact with regular osteocytes and osteoblastic activity.

The authors concluded that a dentin block could be an effective alternative bone graft for alveolar ridge preservation after tooth extraction. It was concluded that dentin block was able to promote new bone formation, without host tissue reactions, and a favorable dentin resorption/bone formation rate [25]. In this case report, we analyzed the clinical, histological, and tomographic results of new bone formation that were obtained using DFDBA and dentin graft for ridge preservation after multiple extractions. Tooth extraction is an ongoing surgical procedure after diverse pathologies such as dental caries, pulp and periapical lesions, periodontal disease, or non-restorable teeth. Therefore, it is of the utmost importance to reduce bone resorption with ridge preservation techniques using biomaterials. The autogenous dentin graft is an alternative for ridge preservation. Previous publications have reported that human dentin is a unique and absorbable matrix with osteoinductive properties, and it may be used as a graft because it releases morphogenetic proteins such as BMP-2. These proteins function as a scaffold for cells that induce bone formation [10]-[13], [21], [23].

In this case report, we used a modified protocol according to Binderman [18], in which dentin particles of 300-1200 $\mu \mathrm{m}$ were obtained in approximately 15-20 minutes. A splitmouth clinical study design was conducted to compare clinical, tomography imaging, and histological outcomes after application of the dentin graft in combination with DFDBA for ridge preservation [22]. The clinical results in this case report showed soft tissue healing with appropriate hydration, color, and texture for both grafts. DFDBA showed a more consistent bone formation without evidence of bone graft particles. For the dentin graft, the sample demonstrated a more disperse distribution, and dentin particles were observed.

In addition to the clinical results from both biomaterials, bone density was analyzed using a CBCT in the premolar area at the 6-month follow-up. In the maxillary area, DFDBA and the dentin graft showed a bone density of 585 HU and 304 $\mathrm{HU}$, respectively. For the mandibular zone, the bone density was $583 \mathrm{HU}$ for the DFDBA and $712 \mathrm{HU}$ for the dentin graft. Recently, Pang et al. [24] analyzed the clinical and histological effects of the autogenous tooth graft material compared to that of inorganic bovine bone in post-extraction alveolar bone augmentation. They showed no infection of graft material, and a vertical proportion of alveolar bone was augmented at 6 months after tooth extraction, which suggests that autogenous tooth graft material is a viable option for alveolar bone ridge following dental extraction.

A biopsy was performed to evaluate bone formation using H\&E and Masson's trichrome stains. When DFDBA was used, mature bone and new bone both showed the presence of osteocytes within lacunae and connective tissue. In the dentin autogenous graft sample, dentin particles were identified as well as associated new bone matrix production within the particles.

Moreover, vascularized connective tissue was identified between bone spaces and dentin particles with a fusion zone and bone matrix production between two particles of mineralized dentin. Ramanauskaite et al. [26] published a systematic review to analyze the clinical evidence of the advantages of using autogenous teeth to reconstruct alveolar ridge defects. This study showed that the clinical evidence is limited, and there are many studies with minor more samples 
from patients and short periods that were evaluated, but the potential of autogenous teeth could be a suitable alternative as a biomaterial substitute to reconstruct alveolar ridge defects.

Autogenous dentin grafts can be considered to be an alternative for alveolar ridge preservation, and they promote the formation of new bone as well as its non-immunogenic, osteoconductive and osteoinductive properties after tooth extraction.

\section{CONCLUSION}

Because of its osteoconductive and osteoinductive properties, autogenous dentin has demonstrated positive results in cases where tooth extraction and ridge preservation are required. An autogenous dentin graft can be considered to be an alternative for bone fill due to its autogenous nature that promotes new bone formation and its non-immunogenic properties. The processing technique proposed by Binderman provides a high-volume graft that is free of bacteria, viruses, and fungi. After its application, histological characteristics that were similar to bone tissue were observed, and there was also an appropriate bone density along with favorable clinical, histological, and tomography imaging outcomes following tooth extraction. Further clinical, histological, and tomographic studies are required to evaluate the long-term results.

\section{REFERENCES}

[1] Khanijou M, Seriwatanachai D, Boonsiriseth K, Suphangul S, Pairuchvej V, Srisatjaluk RL, et al. Bone graft material derived from extracted tooth: A review literature. Journal of Oral and Maxillofacial Surgery, Medicine, and Pathology. 2019;31(1):1-7.

[2] Schropp L, Wenzel A, Kostopoulos L, Karring T. Bone healing and soft tissue contour changes following single-tooth extraction: a clinical and radiographic 12-month prospective study. Int $\mathrm{J}$ Periodontics Restorative Dent. 2003;23(4):313-23.

[3] Tan WL, Wong TL, Wong MC, Lang NP. A systematic review of post-extractional alveolar hard and soft tissue dimensional changes in humans. Clin Oral Implants Res. 2012;23 Suppl 5:1-21.

[4] Leblebicioglu B, Salas M, Ort Y, Johnson A, Yildiz VO, Kim D-G, et al. Determinants of alveolar ridge preservation differ by anatomic location. Journal of Clinical Periodontology. 2013;40(4):387-95.

[5] Klijn RJ, Meijer GJ, Bronkhorst EM, Jansen JA. A meta-analysis of histomorphometric results and graft healing time of various biomaterials compared to autologous bone used as sinus floor augmentation material in humans. Tissue Eng Part B Rev. 2010;16(5):493-507.

[6] Cha JK, Joo M-J, Yoon S, Lee J-S, Choi S-H, Jung U-W. Sequential healing of onlay bone grafts using combining biomaterials with crosslinked collagen in dogs. Clinical Oral Implants Research. 2017;28(1):76-85.

[7] Wei L, Miron RJ, Shi B, Zhang Y. Osteoinductive and Osteopromotive Variability among Different Demineralized Bone Allografts. Clinical Implant Dentistry and Related Research. 2015;17(3):533-42.

[8] Kim YK, Lee J, Um IW, Kim KW, Murata M, Akazawa T, et al. Tooth-derived bone graft material. J Korean Assoc Oral Maxillofac Surg. 2013;39(3):103-11.

[9] Bang G, Urist MR. Bone induction in excavation chambers in matrix of decalcified dentin. Arch Surg. 1967;94(6):781-9.

[10] Kim YK, Kim SG, Yun PY, Yeo IS, Jin SC, Oh JS, et al. Autogenous teeth used for bone grafting: a comparison with traditional grafting materials. Oral Surg Oral Med Oral Pathol Oral Radiol. 2014;117(1): e39-45.

[11] Nampo T, Watahiki J, Enomoto A, Taguchi T, Ono M, Nakano H, et al. A new method for alveolar bone repair using extracted teeth for the graft material. J Periodontol. 2010;81(9):1264-72.

[12] Huggins C, Wiseman S, Reddi AH. Transformation of fibroblasts by allogeneic and xenogeneic transplants of demineralized tooth and bone. J Exp Med. 1970;132(6):1250-8.

[13] Yeomans JD, Urist MR. Bone induction by decalcified dentine implanted into oral, osseous and muscle tissues. Arch Oral Biol. 1967;12(8):999-1008.

[14] Kim Y-K, Kim S-G, Byeon J-H, Lee H-J, Um I-U, Lim S-C, et al. Development of a novel bone grafting material using autogenous teeth. Oral Surgery, Oral Medicine, Oral Pathology, Oral Radiology and Endodontics. 2010;109(4):496-503.

[15] Kim YK, Kim SG, Oh JS, Jin SC, Son JS, Kim SY, et al. Analysis of the inorganic component of autogenous tooth bone graft material. J Nanosci Nanotechnol. 2011;11(8):7442-5.

[16] Kim YK, Lee J, Um IW, Kim KW, Murata M, Akazawa T, et al. Tooth-derived bone graft material. J Korean Assoc Oral Maxillofac Surg. 2013;39(3):103-11.

[17] Hanamura H, Higuchi Y, Nakagawa M, Iwata H, Nogami H, Urist MR. Solubilized bone morphogenetic protein (BMP) from mouse osteosarcoma and rat demineralized bone matrix. Clin Orthop Relat Res. 1980(148):281-90.

[18] Binderman I, Hallel G, Nardy C, Yaffe A, Sapoznikov L. A Novel Procedure to Process Extracted Teeth for Immediate Grafting of Autogenous Dentin. J Interdiscipl Med Dent Sci. 2014;2:154.

[19] Lee S, Gantes B, Riggs M, Crigger M. Bone density assessments of dental implant sites: 3 . Bone quality evaluation during osteotomy and implant placement. Int J Oral Maxillofac Implants. 2007;22(2):20812.

[20] Norton MR, Gamble C. Bone classification: an objective scale of bone density using the computerized tomography scan. Clinical Oral Implants Research. 2001;12(1):79-84.

[21] Murata M, Akazawa T, Mitsugi M, Arafat M, Um I-W, Minamida Y, et al. Autograft of Dentin Materials for Bone Regeneration. Advances in Biomaterials Science and Biomedical Applications2013.

[22] Lesaffre E, Garcia Zattera M-J, Redmond C, Huber H, Needleman I, Dentistry obotISo. Reported methodological quality of split-mouth studies. Journal of Clinical Periodontology. 2007;34(9):756-61.

[23] Ike M, Urist MR. Recycled dentin root matrix for a carrier of recombinant human bone morphogenetic protein. J Oral Implantol. 1998;24(3):124-32.

[24] Pang KM, Um IW, Kim YK, Woo JM, Kim SM, Lee JH. Autogenous demineralized dentin matrix from extracted tooth for the augmentation of alveolar bone defect: a prospective randomized clinical trial in comparison with anorganic bovine bone. Clin Oral Implants Res. 2017 Jul;28(7):809-815. doi: 10.1111/clr.12885. Epub 2016 Jun 8. PMID: 27279547.

[25] Andrade C, Camino J, Nally M, Quirynen M, Martínez B, Pinto N. Combining autologous particulate dentin, L-PRF, and fibrinogen to create a matrix for predictable ridge preservation: a pilot clinical study. Clin Oral Investig. 2020 Mar;24(3):1151-1160. doi: 10.1007/s00784-019-02922-Z. Epub 2019 Jul 10. PMID: 31292776.

[26] Ramanauskaite A, Sahin D, Sader R, Becker J, Schwarz F. Efficacy of autogenous teeth for the reconstruction of alveolar ridge deficiencies: a systematic review. Clin Oral Investig. 2019 Dec;23(12):4263-4287. doi: 10.1007/s00784-019-02869-1. Epub 2019 Mar 11. PMID: 30859329. 